\title{
Causal Oscillations in Cavities with Metallic Surfaces
}

\section{Fatih Erden}

Abstract - Causal oscillations are considered in a cavity with a lossy metallic surface excited by a given external signal of finite duration. No other restrictions on the time dependence of the external signal is needed, except its integrability. A novel format of Maxwell equations in the International System of Units is used for solving the problem.

\section{Introduction}

This study addressed the problem of electromagnetic oscillations in a lossy metallic cavity without resorting to the Fourier and Laplace transforms by working directly in the time domain via the evolutionary approach to electromagnetics (EAE) in accordance with the principle of causality [1]. The supposition of the time harmonic theory that all fields sinusoidally vary in time eliminates the time derivative from the original Maxwell's equations (MEs), and the solutions to the elliptic partial differential equations do not satisfy the causality principle. The time dependence is suppressed throughout the time harmonic cavity analyses, where the field and source quantities have a time variation of $\exp (-i \omega t)[2]$.

Detailed background information on interior electromagnetic problems and the analytical time domain solution of cavity fields can be found in [3, 4]. Some of the publications, cited in the references, are intended to direct the readers to further information concerning the possibilities of the EAE for solving transient processes excited by various signals in perfectly conducting cavities filled with different sorts of media [5-10].

The cavity under study is bounded by a closed, singly connected lossy metallic surface $S$ that is considered as a smooth enough one. The term smooth enough implies that none of the possible inner angles of $S$ (i.e., measured within $S$ ) exceeds $\pi$. For example, the surface of a rectangular cavity is smooth enough in that sense. Denote the outward normal to $S$ and tangential to $S$ unit vectors as $\mathbf{n}$ and $\mathbf{l}$, respectively. The boundary $S$ of cavity volume $V$ has the properties of a wellconducting metal parametrized by its surface impedance s. Over $S$, the Leontovich boundary condition (LBC) holds as

$$
[\mathbf{n} \times \mathcal{E}(\mathbf{r}, t)]=\varsigma \mathbf{l} \cdot \mathcal{H}(\mathbf{r}, t), \mathbf{r} \in S
$$

and relates to the tangential to $S$ components of the

Manuscript received 5 July 2021.

Fatih Erden is with the National Defense University, National Defense University, Naval Academy, Istanbul 34940, Turkey; email: ferden@dho.edu.tr. fields $\mathcal{E}_{\ell}=\mathbf{n} \times \mathcal{E}$, and $\mathcal{H}_{\ell}=\mathbf{l} \cdot \mathcal{H}$. The LBC is accurate when the permittivity of metal is large but finite [11]. The standard fields $\mathcal{E}$ and $\mathcal{H}$ in [1] have the dimensions in the International System of Units (SI) as volt per meter $\lfloor\mathrm{V} / \mathrm{m}\rfloor$ and ampere per meter $\lfloor A / \mathrm{m}\rfloor$, respectively. The impedance $\varsigma$ has dimension of ohm $\lfloor\Omega\rfloor$.

The format (1) is somewhat inconvenient for solving the titled boundary value problem because $\mathcal{E}_{\ell}, \mathcal{H}_{\ell}$, and $\varsigma$ have distinct dimensions. To simplify the boundary condition (1), two scaling factors $\varepsilon_{0}^{\mathrm{V}}$ and $\mu_{0}^{\mathrm{A}}$ for the fields $\mathcal{E}$ and $\mathcal{H}$ are used. They were defined as

$$
\varepsilon_{0}^{\mathrm{V}} \triangleq \sqrt{\frac{1 N}{\varepsilon_{0}}}\left\lfloor\mathrm{~V}=\frac{N \mathrm{~m}}{A s}\right\rfloor, \mu_{0}^{\mathrm{A}} \triangleq \sqrt{\frac{1 N}{\mu_{0}}}\lfloor A\rfloor
$$

where $(1 \mathrm{~N})$ under the radicals implies a force of 1 Newton and $\varepsilon_{0}$ and $\mu_{0}$ are the permittivity and permeability of free space, specified in SI units $[12,13]$. Scaling the fields yields

$$
\underbrace{\mathcal{E}(\mathbf{r}, t)}_{\lfloor\mathrm{V} / \mathrm{m}\rfloor}=\underbrace{\varepsilon_{0}^{\mathrm{V}}}_{\lfloor\mathrm{V}\rfloor} \underbrace{\mathbb{E}(\mathbf{r}, t)}_{\lfloor 1 / \mathrm{m}\rfloor}, \underbrace{\mathcal{H}(\mathbf{r}, t)}_{\lfloor\mathrm{A} / \mathrm{m}\rfloor}=\underbrace{\mu_{0}^{\mathrm{A}}}_{\lfloor\mathrm{A}\rfloor} \underbrace{\mathbb{H}(\mathbf{r}, t)}_{\lfloor 1 / \mathrm{m}\rfloor} .
$$

where $\mathbb{E}$ and $\mathbb{H}$ are new field vectors sought for, $\mathbf{r}$ is position vector of a point of observation, and $t$ is an observation time. Substituting the scaled fields in (1) results in a novel format of the $\mathrm{LBC}$ as

$$
\mathbf{n} \times \mathbb{E}(\mathbf{r}, t)=\alpha \mathbf{l} \cdot \mathbb{H}(\mathbf{r}, t), \mathbf{r} \in S
$$

where $\alpha=\hat{\zeta} \hat{\rho}$ is a small dimension-free parameter. Also, $\hat{\zeta}$ is the numeric value of the given impedance, and the small constant $\hat{\rho}=2.654 \times 10^{-3}$ is the numeric value of $\rho=\mu_{0}^{\mathrm{A}} / \varepsilon_{0}^{\mathrm{v}}$. The dimensional balance in (4) is evident, as opposed to (1).

The goal of this article is to study the evolution of the oscillations in the cavity described previously. A given external signal has a beginning in time and may have a finite duration. No other restrictions on the time dependence of the external signal are imposed, except its integrability.

The composition of the article is as follows: In Section 2, a statement of the initial-boundary value problem (IBVP) is given where the LBC (4) participates. The novel format of MEs is used $[12,13]$. In that format, the fields $\mathbb{E}$ and $\mathbb{H}$ are the same as in (4). In Section 3, the modal basis is derived, which is applicable for the modal decompositions of the cavity fields $\mathbb{E}(\mathbf{r}, t)$ and $\mathbb{H}(\mathbf{r}, t)$, with the time-dependent modal amplitudes. In Section 4, the Cauchy problem is obtained for the modal amplitudes, and several examples of the causal evolution of the forced cavity 
oscillations are exhibited graphically. In Section 5, the main results are listed and discussed.

\section{Initial-Boundary Value Problem}

Standard MEs in SI units are overloaded by individual physical dimensions, which the fields $\mathcal{E}$ and $\mathcal{H}$ have in this format, and the dimensional coefficients $\varepsilon_{0}$ and $\mu_{0}$ are involved herein. A novel format of MEs in SI units has been published recently in $[12,13]$

$$
\begin{aligned}
& \nabla \times \mathbb{H}(\mathbf{r}, t)=\frac{\partial}{c \partial t} \mathbb{E}(\mathbf{r}, t)+\mathbb{J}(\mathbf{r}, t) \\
& -\nabla \times \mathbb{E}(\mathbf{r}, t)=\frac{\partial}{c \partial t} \mathbb{H}(\mathbf{r}, t)
\end{aligned}
$$

for the problem under study. Note that the field vectors and also space and time derivatives (i.e., $\nabla \times$ and $\frac{\partial}{c \partial t}$, where $c$ is the speed of light) have the common dimension of $\lfloor 1 / \mathrm{m}\rfloor$. The given current density, $\mathbb{J}(\mathbf{r}, t)$, which is responsible for inducing forced oscillations in the cavity, should have dimension of $\left|1 / \mathrm{m}^{2}\right|$. The dimensional balance in (5) is evident as opposed to the standard format of MEs in SI units.

The MEs with the time derivative belong to the hyperbolic type of PDE. Therefore, they should be supplemented with the initial conditions (for $t=0$ ) that may be chosen as

$$
\mathbb{E}(\mathbf{r}, 0)=\mathbf{0} \text { and } \mathbb{H}(\mathbf{r}, 0)=\mathbf{0}
$$

The differential equations in (5) hold in the open domain $V$ (i.e., where $\mathbf{r} \in V$ but $\mathbf{r} \notin S$ ). Over the boundary $S$, the MEs have an algebraic form of the boundary condition, as (4). We are free to specify the external force function $\mathbb{J}(\mathbf{r}, t)$ as

$$
\mathbb{J}(\mathbf{r}, t)=\mathbf{0}, \text { while } t<0
$$

Specifying $\mathbb{J}(\mathbf{r}, t)$ for $t>0$ will be given afterward.

\section{Modal Basis and Modal Decompositions}

The Hilbert space $\mathfrak{I}_{2}$ is chosen as the space of solution to the IBVP under study. The inner product for a pair of vectors from $\mathfrak{L}_{2}(\mathbf{A}(\mathbf{r})$ and $\mathbf{B}(\mathbf{r}))$ is

$$
\langle\mathbf{A}, \mathbf{B}\rangle=\frac{1}{V} \int_{V} \mathbf{A}(\mathbf{r}) \cdot \mathbf{B}^{*}(\mathbf{r}) d V
$$

where the star at $\mathbf{B}^{*}$ implies complex conjugation of $\mathbf{B}$.

\subsection{Separation of a Self-Adjoint Operator From MEs}

Introduce an operator $\Re$, which acts on the auxiliary six-component column vectors chosen as $\mathfrak{X}(\mathbf{r})=\operatorname{col}(\mathbf{E}(\mathbf{r}), \mathbf{H}(\mathbf{r}))$, varying in the closed domain $V$ (i.e., where $\mathbf{r} \in V$ and $\mathbf{r} \in S$ ). In the open domain, operator $\Re$ acts on the vectors $\mathfrak{X}(\mathbf{r})$ as the $6 \times 6$ matrix procedure $\Re^{\prime}$ specified in (9) where $\mathcal{O}$ is $3 \times 3$ zerovalued matrix. The differential procedure $\Re^{\prime}$ is composed of the operation $\nabla \times$, taken from the left-hand side MEs (5). The imaginary unit $i=\sqrt{-1}$ is installed in $\Re^{\prime}$ heuristically. Over the boundary $S$, the operator $\Re$ acts as the perfect boundary conditions specified as,

$$
\begin{gathered}
\Re^{\prime} \mathfrak{X}(\mathbf{r})=\underbrace{\left(\begin{array}{cc}
\mathcal{O} & \mathfrak{i} \nabla \times \\
-\mathfrak{i} \nabla \times & \mathcal{O} \mathscr{E}
\end{array}\right)}_{\Re^{\prime}} \underbrace{\left(\begin{array}{c}
\mathbf{E}(\mathbf{r}) \\
\mathbf{H}(\mathbf{r})
\end{array}\right)}_{\mathfrak{X}(\mathbf{r})} \\
\mathbf{n} \times \mathbf{E}(\mathbf{r})=\mathbf{0}, \quad \mathbf{n} \cdot \mathbf{H}(\mathbf{r})=0, \quad \mathbf{r} \in S
\end{gathered}
$$

The operator $\Re$ is self-adjoint in the solution spaces that was proven in [1]. Hence, the operator eigenvalue equation for the operator $\Re$ holds as

$$
\Re \mathfrak{X}_{\mathfrak{n}}(\mathbf{r})=k_{n} \mathfrak{X}_{\mathfrak{n}}(\mathbf{r}) \text { where } n=1,2, \ldots
$$

Herein, $k_{n}$ are the eigenvalues of the operator $\Re$. As long as this operator has a physical dimension of $\lfloor 1 / \mathrm{m}\rfloor$ (5), the eigenvalues $k_{n}$ have the same dimension as well. The spectrum of eigenvalues $\left(k_{n}, n=1,2, \ldots\right)$ is countable because the domain $V$ is finite. The eigenvectors are linearly independent and compose a complete set in $\mathbb{Z}_{2}$ in total [14]. The topology of $\mathbb{Z}_{2}$ has been studied in [1]. The space $\Omega_{2}$ is presentable via a direct sum of four subspaces. Two of these subspaces involve the solenoidal eigenvectors only. The latter correspond to the eigenvalues $k_{n} \neq 0$, and the fragments satisfy two conditions as $\nabla \cdot \mathbf{E}_{n}=0$ and $\nabla \cdot \mathbf{H}_{n}=0$. The other two subspaces involve the irrotational eigenvectors, each of which only corresponds to the eigenvalue $k_{0}=0$ and satisfies either the condition $\nabla$ $\times \mathbf{E}_{n}=0$ or the condition $\nabla \times \mathbf{H}_{n}=0$.

\subsection{A Basis for the Solenoidal Eigenvectors}

Analysis of the operator eigenvalue (11) shows that the elements $\mathbf{E}_{n}(\mathbf{r})$ and $\mathbf{H}_{n}(\mathbf{r})$ of the solenoidal eigenvectors satisfy two sets of the equations simultaneously with the boundary conditions as

$$
\left.\begin{array}{l}
\nabla \times \mathbf{H}_{n}=-i k_{n} \mathbf{E}_{n}, \nabla \cdot \mathbf{H}_{n}=0,\left.\mathbf{n} \cdot \mathbf{H}_{n}\right|_{S}=0 \\
\nabla \times \mathbf{E}_{n}=i k_{n} \mathbf{H}_{n}, \nabla \cdot \mathbf{E}_{n}=0, \mathbf{n} \times\left.\mathbf{E}_{n}\right|_{S}=\mathbf{0}
\end{array}\right\}
$$

The conditions of orthonormalization hold as

$$
\left.\begin{array}{c}
\left\langle\mathbf{E}_{n^{\prime}}, \mathbf{E}_{n}\right\rangle=\frac{1}{V} \int_{V} \mathbf{E}_{n^{\prime}} \cdot \mathbf{E}_{n}^{*} d V=\delta_{n^{\prime} n} \\
\left\langle\mathbf{H}_{n^{\prime}}, \mathbf{H}_{n}\right\rangle=\frac{1}{V} \int_{V} \mathbf{H}_{n^{\prime}} \cdot \mathbf{H}_{n}^{*} d V=\delta_{n^{\prime} n}^{*}
\end{array}\right\}
$$

where $\delta_{n^{\prime} n}$ is Kronecker's delta.

\subsection{Modal Expansions of the Time-Domain Fields}

The six-component solenoidal column vector is

$$
\mathfrak{X}(\mathbf{r}, t)=\operatorname{col}(\mathbb{E}(\mathbf{r}, t), \mathbb{H}(\mathbf{r}, t))
$$

Its three-component fragments $\mathbb{E}$ and $\mathbb{H}$ sought for are presented via the modal field decomposition as

$$
\left.\begin{array}{l}
\mathbb{E}(\mathbf{r}, t)=\sum_{n^{\prime}=1}^{\infty} e_{n^{\prime}}(t) \mathbf{E}_{n^{\prime}}(\mathbf{r}) \\
\mathbb{H}(\mathbf{r}, t)=\sum_{n^{\prime}=1}^{\infty} h_{n^{\prime}}(t) \mathbf{H}_{n^{\prime}}(\mathbf{r})
\end{array}\right\}
$$




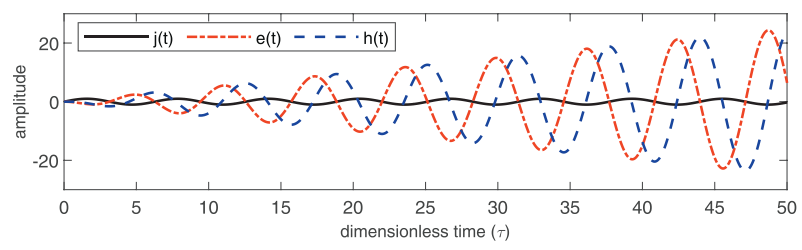

Figure 1. $\beta_{n}=0, j(t)=\sin \left(t c k_{n}\right)$, where $t \geq 0$.

where the basis elements $\mathbf{E}_{n^{\prime}}$ and $\mathbf{H}_{n^{\prime}}$ may already be known, and the amplitudes $e_{n^{\prime}}$ and $h_{n^{\prime}}$ are the sought for ones. The current density $\mathbb{J}(\mathbf{r}, t)(5)$ is presented as

$$
\mathbb{J}(\mathbf{r}, t)=j(t) \mathbf{I}(\mathbf{r})
$$

where $j(t)$ is a given signal. The three-component vector $\mathbf{I}(\mathbf{r})$ is responsible for supplying the given signal $j(t)$ to the cavity. The vector $\mathbf{I}(\mathbf{r})$ is specified by an input item, which supplies the signal to the cavity volume.

$$
\mathbf{I}(\mathbf{r})=\sum_{n^{\prime}=1}^{\infty} g_{n^{\prime}} k_{n^{\prime}} \mathbf{E}_{n^{\prime}}(\mathbf{r})
$$

where $g_{n^{\prime}}$ are dimension-free constants, which specify a position of the input item within $V$ and $k_{n^{\prime}}$ are the eigenvalues of $\Re$, specifying the solenoidal modes.

\section{Evolutionary Equations for the Amplitudes}

Projecting MEs (5) onto the basis (12) is executed via the procedure of inner product (8) as follows:

$$
\begin{aligned}
& \left\langle\nabla \times \mathbb{H}(\mathbf{r}, t), \mathbf{E}_{n}(\mathbf{r})\right\rangle=\left\{\begin{array}{c}
\frac{\partial}{c \partial t}\left\langle\mathbb{E}(\mathbf{r}, t), \mathbf{E}_{n}(\mathbf{r})\right\rangle \\
+\left\langle\mathbb{J}(\mathbf{r}, t), \mathbf{E}_{n}(\mathbf{r})\right\rangle
\end{array}\right\} \\
& -\left\langle\nabla \times \mathbb{E}(\mathbf{r}, t), \mathbf{H}_{n}(\mathbf{r})\right\rangle=\frac{\partial}{c \partial t}\left\langle\mathbb{H}(\mathbf{r}, t), \mathbf{H}_{n}(\mathbf{r})\right\rangle
\end{aligned}
$$

The result obtained by using LBC (4) is exhibited as

$$
\begin{gathered}
\left\langle[\nabla \times \mathbb{H}] \cdot \mathbf{E}_{n}\right\rangle=i k_{n}\left\langle\mathbb{H} \cdot \mathbf{H}_{n}\right\rangle+i k_{n} g_{n} j(t)\left\langle\mathbf{I}, \mathbf{E}_{n}\right\rangle \\
-\left\langle[\nabla \times \mathbb{E}], \mathbf{H}_{n}\right\rangle=-\beta_{n} h_{n}(t)+i k_{n}\left\langle\mathbb{E}, \mathbf{E}_{n}\right\rangle \\
\beta_{n}=\alpha \frac{1}{S} \int_{S} \mathbf{H}_{\ell n} \cdot \mathbf{H}_{\ell n}^{*} d S
\end{gathered}
$$

where $\beta_{n}$ is a lossy parameter and $\mathbf{H}_{\ell n}$ is tangential to $S$ component of the field $\mathbf{H}_{n}$ from (12). The inner products placed at the left-hand sides in (18) and (19) are calculated by an analogous technique given in [11, pp. 557-558). Calculations of the inner products $\left\langle\mathbb{E}, \mathbf{E}_{n}\right\rangle$ and $\left\langle\mathbb{H}, \mathbf{H}_{n}\right\rangle$ at the right-hand sides are elementary, substituting the modal field expansions (15). In total,

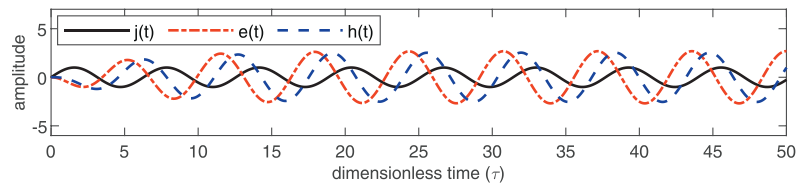

Figure 2. $\beta_{n}=0.2, j(t)=\sin \left(t c k_{n}\right)$, where $t \geq 0$.

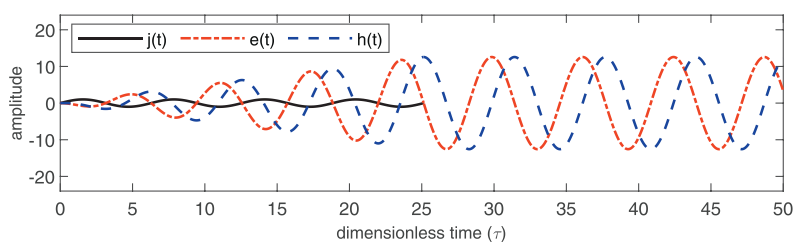

Figure 3. $\beta_{n}=0, j(t)=\sin \left(t c_{n}\right)$, where $0 \leq t \leq T$.

(18)-(22) result in the Cauchy problem for the modal amplitudes $e_{n}(t)$ and $h_{n}(t)$, as

$$
\begin{gathered}
\frac{d}{c d t} e_{n}(t)-i k_{n} h_{n}(t)=-j(t) \\
\frac{d}{c d t} h_{n}(t)+\beta_{n} h_{n}(t)-i k_{n} e_{n}(t)=0 \\
e_{n}(0)=0 \text { and } h_{n}(0)=0
\end{gathered}
$$

where the initial conditions, which follow from (6), are added. The exact analytical causal solutions are obtained by the method of matrix exponential [1], and the graphical results are exhibited in the following.

The dimensionless time $\tau$ is specified as $\tau=t c k_{n}$, where $c$ is the speed of light and $k_{n}$ is the eigenvalue of the self-adjoint operator $\Re$. The eigenfrequencies of the cavity modes for the lossy cavity are $\omega_{n}=c k_{n} \sqrt{1-\beta_{n}^{2}}$, where $\beta_{n}$ is the lossy parameter. If the cavity surface is perfectly conducted (i.e., $\beta_{n}=0$ ), then $\omega_{n} \equiv \dot{\omega}_{n}=c k_{n}$. If $\beta_{n}=0$ and the signal is unbounded (see Figure 1), the modal amplitudes $e_{n}$ and $h_{n}$ increase linearly in time. This is resonance. If $\beta_{n} \neq 0$, the amplitudes $e_{n}$ and $h_{n}$ are proportional to $\left[-\exp \left(-\beta_{n} \tau\right)\right]$ at the beginning of the oscillations (i.e., when $\tau \geq 0$ ) (see Figure 2). If the signal is turned off (i.e., $\tau \geq T$ ) (see Figure 3 and 4), then the amplitudes $e_{n}$ and $h_{n}$ decay proportionally to $\exp \left(-\beta_{n} \tau\right)$ for the lossy cavity.

\section{Main Results}

The modal basis for the time domain studies is derived without supposing that the cavity fields are time harmonic. To this aim, a self-adjoint operator is extracted from the system of MEs (5), and the operator eigenvalue equation (11) is derived and solved.

The modal field expansions for the fields $\mathbb{E}(\mathbf{r}, t)$ and $\mathbb{H}(\mathbf{r}, t)$ are obtained with time-dependent modal amplitudes, and the Cauchy problem is obtained and solved for the amplitudes. The evolution of the modal

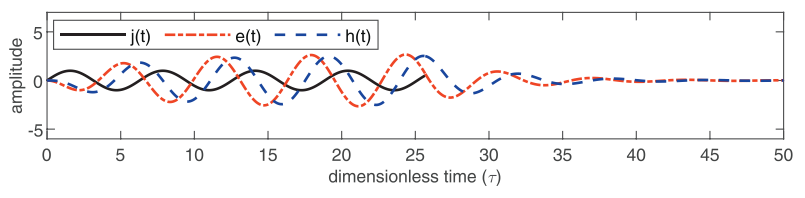

Figure 4. $\quad \beta_{n}=0.2, j(t)=\sin \left(t c k_{n}\right)$, where $0 \leq t \leq T$. 
amplitudes, which are excited in the cavity by a given signal of finite duration is exhibited graphically.

\section{Acknowledgments}

This work was supported, in part, by the Scientific and Technological Research Council of Turkey (project 120E390). The author is grateful to project advisor, Oleg A. Tretyakov, for making valuable suggestions and reviewing the manuscript. The author thanks the anonymous reviewers and Editor-in-Chief, Piergiorgio L. E. Uslenghi, for helpful and constructive comments on the article.

\section{References}

1. O. A. Tretyakov, "Essentials of Nonstationary and Nonlinear Electromagnetic Field Theory," in M. Hashimoto, M. Idemen, and O. A. Tretyakov (eds.), Analytical and Numerical Methods in Electromagnetic Wave Theory, Tokyo, Science House Co., 1993, Chapter 3.

2. D. A. Hill, Electromagnetic Fields in Cavities: Deterministic and Statistical Theories, Piscataway, NJ, WileyIEEE Press, 2009.

3. W. Geyi, Foundations of Applied Electrodynamics, New York, Wiley, 2010.

4. W. Geyi, "Time-Domain Theory of Metal Cavity Resonator," PIER, 78, September 2008, p. 219-253, doi: 10.2528/PIER07090605

5. S. Aksoy and O. A. Tretyakov, "Study of a Time Variant Cavity System," Journal of Electromagnetic Waves and Applications, 16, April 2012, pp. 1535-1553, doi: 10. 1163/156939302X00985.

6. S. Aksoy and O. A. Tretyakov, "The Evolution Equations in Study of the Cavity Oscillations excited by a Digital Signal," IEEE Transactions on Antennas and Propagation, 52, 1, January 2004, pp. 263-270, doi: 10.1109/TAP. 2003.822399.

7. S. Aksoy, M. Antyufeyeva, E. Basaran, A. A. Ergin, and O. A. Tretyakov, "Time-Domain Cavity Oscillations Supported by a Temporally Dispersive Dielectric," IEEE Transactions on Microwave Theory and Techniques, 53, 8, August 2005, pp. 2465-2471, doi: 10.1109/TMTT. 2005.852784 .

8. O. A. Tretyakov and F. Erden, "Temporal Cavity Oscillations Caused by a Wide-Band Waveform," PIER $B$, 6, April 2008, pp. 183-204, 10.2528/PIERB08031222.

9. F. Erden and O. A. Tretyakov, "Excitation of the RealValued Electromagnetic Fields in a Cavity by a Given Transient Signal," Physical Review E, 77, 5, May 2008, p. 056605, doi: 10.1103/PhysRevE.77.056605.

10. F. Erden, "Evolutionary Approach to Solve a Novel Time-Domain Cavity Problem," IEEE Transactions on Antennas and Propagation, 65, 11, November 2017, pp. 5918-5931, doi: 10.1109/TAP.2017.2752240.

11. I. N. Toptygin, Electromagnetic Phenomena in Matter: Statistical and Quantum Approaches, Weinheim, Germany, Wiley-VCH, 2015.

12. O. A. Tretyakov and F. Erden, "A Novel Simple Format of Maxwell's Equations in SI Units," IEEE Access, 9, June 2021, p. 88272, doi: 10.1109/Access.2021.3089673.

13. O. A. Tretyakov, O. Butrym, and F. Erden, "Innovative Tools for SI Units in Solving Various Problems of Electrodynamics," in K. Kobayashi and P. Smith (eds.), Advances in Mathematical Methods for Electromagnetics, London, IET, 2021, Chapter 27.

14. H. Weyl, "The Method of Orthogonal Projection in Potential Theory," Duke Mathematical Journal, 7, 1, 1940, pp. 411-444. 